\title{
Automated Tool Selection for Computer-Aided Process Planning in Sheet Metal Bending
}

\author{
J. R. Duflou ${ }^{1}$ (2), T. H. M. Nguyen ${ }^{1}$, J.-P. Kruth ${ }^{1}$ (1), D. Cattrysse ${ }^{2}$ \\ ${ }^{1}$ Department of Mechanical Engineering, ${ }^{2}$ Centre for Industrial Management \\ Katholieke Universiteit Leuven, Celestijnenlaan 300, 3001 Leuven, Belgium
}

\begin{abstract}
Bend sequencing and tool selection have long been the main hurdles for achieving automatic process planning for sheet metal bending. In this unique process, the complex shape and position transitions of workpieces make it hard to obtain a collision-free operation plan. The time-consuming involvement of human experts is often required to solve more complex problems. This paper presents a tool selection methodology to be integrated in the automatic bend sequencing system discussed in previous work, therefore contributing to fully automated process planning for bent sheet metal parts. Both the described selection strategy and the related algorithms have been implemented in an industrial software package. The results presented in this paper, as illustrated by a number of case studies, demonstrate that automatic process planning for sheet metal bending is feasible and that the developed system provides well-optimised solutions with a reasonable time complexity.
\end{abstract}

Keywords:

CAPP, Tooling, Bending

\section{INTRODUCTION}

Sheet metal bending using press brakes is a flexible process for parts formed from metal sheets by linear bends. Process planning for this process includes two interlacing aspects, namely bend sequencing and tool selection. The first aspect tackles the method to sequence all the bending operations in order to avoid collisions between the part being bent and other objects in the production environment. Efforts to automate this step resulted in computer aided solutions, including heuristic search techniques based on a rule set for collision avoidance $[1,2,3]$, and on tolerance specifications $[4,5]$. However, such approaches often rely on interactive tool selection or do not take tool selection into consideration. Consequently, if the tools are not strategically selected by experienced process planners, feasible solutions often cannot be assured.

In parallel, tool selection has been mentioned or partially dealt with by researchers in the field $[6,7,8,9]$. In consideration of the macro technological aspects, an expert system was built using LISP language to interactively aid process planners in choosing the right sheet metal process and tooling type [7,9]. In a more detailed perspective, existing automatic tool selection strategies $[6,8]$ often start from a predetermined bending sequence. Such strategies frequently result in expensive construction of specially tailored tools, which are needed to avoid obvious collisions that result from the chosen bend sequence. Traditionally, the approach chosen to eliminate the need for expert-based tool selection is a combinatorial search problem formulation for an open selection [2, 6]. Under such regime, solutions for complex parts cannot be identified within a limited time span.

This research presents a methodology to automatically identify feasible and well-optimised tool sets based on part descriptions and available production environments. The method can be integrated in and is complementary to existing bend sequencing procedures. Feasible tools are selected based on technological and geometric considerations. The latter is implemented into two steps: preselection based on the final part description and refined selection based on intermediate stages of the workpiece during bending. Additionally, common guidelines for optimal tool usage are translated into optimisation strategies, ready to be used in different process planning phases. The methodology presented is demonstrated by an industrial software implementation. Test results from benchmark parts support the conclusions on the feasibility and performance of the method.

\section{TOOL SELECTION METHODOLOGY}

Tool selection methods for production processes typically consist of procedures to convert product specifications into selection criteria for related parameters, in order to identify the relevant machines and tools. Moreover, where a minimal production resource is desired, optimisation algorithms are usually applied. These two aspects are collectively handled in the feasible tool selection and optimisation procedures for bent sheet metal products as explained below.

\subsection{Feasible tool selection}

\section{Technological considerations}

Similar to other production processes, tools selected for sheet metal bending should meet the technological constraints imposed by the part to be produced in order to assure technical feasibility and to provide the appropriate bend line quality as determined by the design specifications. The relations between these two aspects are found scattered in literature in either tabulated format or as simple rules of thumb. Based on these relations, three subsequent steps are distinguished in this study to preselect tools under technological considerations.

The initial step is to select the bending technique, limited to air bending and bottoming on press brakes. The choice to be made depends on the accuracy, and the bend features required. The information of the bend angle accuracy is converted into requirements for the bending techniques, and considerations for available in-process measuring and adaptive control equipment. Bend lines characterised by special features, such as hemmed edges, are immediately provided with appropriate special tooling in this step.

The second step is to select the machine class, i.e. the range of setup length, tonnage, and gauging requirements. The force required to make the longest bend with the material and bending technique selected is 
calculated in order to specify the tonnage of the machine to be used. Bend lines with no parallel gauging solution imply selection of press brakes with independent back gauges.

The third step is to select the tool class compatible with the chosen bending techniques and machine classes. Decisions are made based on the required force for the bend features as well as their shapes. This step takes as input various parameters of the part, such as the sheet thickness, material properties of the sheet, and geometric characteristics of the bend features to be performed, including the required internal radius, the bend angle, and the minimum bend-flange width.

\section{Geometric considerations}

Studies [2, 3] show that most collisions in bending occur between bent parts and tools, especially when final shapes are nearly achieved. Besides altering the bend sequence, pragmatic solutions to most of those situations are appropriate tool selections by process planners. Therefore, in addition to complying with the technological considerations, tool selection for a bent part must firstly take into account its final shape, and secondly allow necessary adjustments based on its intermediate shapes.

As a result, at the strategic level geometric tool selection can be divided into two phases. The first phase is called the preselection phase, which eliminates obvious collisionprone tool shapes based on the envisaged part shape. Subsequently, bend lines are assigned with corresponding preselected tools, providing a favourable initiation for the bend sequencing step, since it efficiently reduces the number of collisions encountered while searching for a collision-free bend sequence [2]. The second phase is called the refined selection phase, which selects the tools based on the collisions encountered by the part in its intermediate stages during bend sequencing. If the tools are already preselected, refined selection only adjusts the preselected tools to suit the stricter conditions imposed by the collisions encountered.

At the tactical level, an algorithm for linking the geometric aspects between bent parts and bending tools has been developed. Instead of direct matching between available tool types and part shapes, which is not applicable due to the continuous shape transitions of the bent parts [2], a generic rule set has been established in this study to link possible collision patterns with geometric features, representing the collision avoidance capability of tools. To facilitate the application of these rules, in this study each collision pattern is defined by a combination of the following factors: the machine component or tool involved in the collision, the collision flange of the part being bent, the direction from which the collision flange comes, and the bending phase in which the collision occurs. The collision patterns are identified in the preselection phase by analysing the final part; while in the refined selection phase, they are directly identified from the collision detection module activated during bend sequencing [2]. The utilisation of the information encapsulated in collision patterns, instead of pure tool and part geometries, allows an efficient identification of requirements for tool selection by a selective application of rules, a fast estimation of actual collision, and therefore a handy production of criteria for geometric tool selection.

Firstly, the rules qualitatively specify the following feature requirements on tools based on the collision component identified in the collision pattern. For punches, (1) a greater height is required for collisions with the machine ram, (2) a horn tool - for collisions at the side, (3) a gooseneck feature - for collisions along the bend line, (4) tool windowing - for collisions partially along the bend line where no gooseneck tool can be found because the collision flange protrudes too far. Similarly, changing of the die features can give solutions, such as (1) a die with a thinner body width - for collisions with the die, (2) a greater die height - for collisions with the machine table.

Secondly, the range of values to be met by the geometric features specified by the rules above is quantitatively specified from the collision patterns. For each of the patterns encountered, the respective collision flange(s) are clipped based on the collision component's bounding box and the collision direction in order to define the coordinates of the actual collision ranges in 3D.

Afterwards, the derivation of logical constraints for tool selection combines the logical part from the triggered rules and the value range obtained from processing the collision flanges. In this way, appropriate sets of quantified rules are combined for all collision patterns foreseen per bend line. An example of the procedure of tool selection based on the analysis of collision patterns is shown in Figure 1, where the tool parameters used in the constraints generated are explained in Figure 3.

(a) Collision patterns:
Collision with punch from
side +/- 1 of flange 1 and 3
after bend
(b) Actual interference ranges:
Side-1 Lower Bound $X=-10.0000, Y=46.3887, Z=0$
Upper Bound $X=-2.1213, Y=54.9744, Z=97$
Side+1 Lower Bound $X=2.1213, Y=46.3887, Z=0$
Upper Bound $X=10.0000, Y=54.9744, Z=97$

(c) Rule: Choose a gooseneck punch

(d) Logical constraints and corresponding tools selected for the collisions foreseen: GOOSENECK PUNCH

$\{[(C$-side $=-1)$ AND $(C$-depth $\geq-2.1213)$ OR

AND $(C$-low $\leq 46.3887) A N D(C-h i g h \geq 54.9744)]$

$[(C$-side $=+1)$ AND $(C$-depth $\leq 2.1213)$

AND $(C$-low $\leq 46.3887) A N D(C$-high $\geq 54.9744)]\}$

Figure 1: Tool selection based on collision pattern.

In assistance to the application of the rules described above, a thorough analysis on the important threedimensional features of parts and tools has been accomplished in this research to quickly identify potential collision patterns from bent parts and the collision avoidance capability of tools for bending.

On the one hand, analysing three-dimensional part features shows that there are often a number of difficult partitions, commonly called local details, residing in the designs to be produced. Such local details often cause (a combination of) collision patterns, requiring precise tool selection. The definitions and properties of the details identified together with their corresponding collision patterns are shown in Figure 2. If graph representations are used for bent parts and the local details, where nodes and arcs correspond to bend flanges and bend lines respectively, the pattern graphs representing the details can be matched with the target graphs representing parts in polynomial time [10]. Therefore, all the local details can be detected from bent parts, facilitating the identification of their respective collision patterns.

On the other hand, aiming at characterising the collision avoidance capability offered by individual tools in a uniform way, despite their numerous commercial coding and shape variety, a system capable of extracting the geometric parameters representing the collision avoidance capability of tools has been developed. Among all the intrinsic shape features, a vital collision avoidance capability of a punch has been identified in this study and is represented by a typical shape feature, which is referred 
to as a C-feature. As shown in Figure 3.a, the C-feature is a concave shape, and as such provides a free-access space in the tool body, allowing bent flanges to occupy the space without collisions. C-features are generically identified in geometrically described tool profiles by detecting concave vertices on the tool boundaries. A tool can have multiple $\mathrm{C}$-features, residing on either side of the tool. The geometric parameters related to this special shape feature are stored complementary to the actual tool geometry description. This database supports fast queries based on the above-mentioned rules for tool selection. By maintaining the data of the collision avoidance capability of all tools, tool selection infeasibilities are quickly identified to either provide early feedback for design revision in the preselection phase, or for robust pruning of bend sequence solutions during the refined selection phase.

Detail name
and example $\begin{aligned} & \text { Topological } \\ & \text { abstraction }\end{aligned}$ $\begin{aligned} & \text { Collision patterns } \\ & \text { - die, table } \\ & \text { - bent side of } Z \\ & \text { - flanges } 1,3 \\ & \text { - before bending }\end{aligned}$

Figure 2: Local details and collision patterns related.

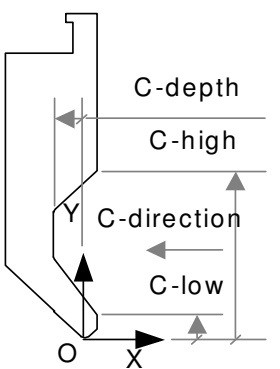

(a)

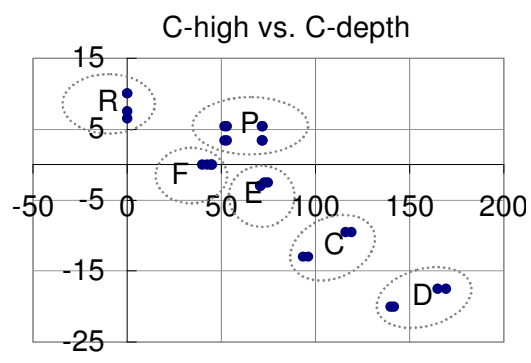

(b)
Figure 3: C-feature (a) description and (b) tool clustering based on this feature.

\subsection{Optimisation of resources}

Minimising the number of required tool stations and limiting changes between tool types have a beneficial effect on the tool setup and changeover times. The following common guidelines are therefore applied during tool selection:

1- Use the same tool or at least the same tool family, i.e. tools having the same height, for the jobs being pooled together.

2- Use the tools that are commonly used for production in the company.
3- Use the most flexible tools as identified from industrial use in case there is no other preference.

These guidelines are implemented in three optimisation strategies, the detailed applications of which are well explained in [11] and can be summarised as follows:

The first strategy assigns a tool to each bend so that the number of tools used is minimal, provided that all the feasible tools are known for all bend lines. Using this strategy, tool sharing between different bend lines is maximised. The implementation of this strategy makes use of a Set Covering Problem (SCP) formulation [12], which can be applied whenever multiple tools are simultaneously defined for single bend lines. This strategy is applied to minimise the preselection set before launching it as input for bend sequencing, or to minimise the final selection set after a collision-free bend sequence is identified.

The second strategy maximises the global preference for the selected tool set. A preference factor is calculated for each tool based on the production planning data available in the manufacturing company. Three elements are taken into account for this purpose: historical tool utilisation frequency data, as obtained from process planning records, tool selection data for other parts in the same list of production tasks, and previous tool allocations to other bend lines in the same part. The preference factor determines the order in which tools are chosen from the list of technically and geometrically feasible solutions. This strategy is applied in all phases of tool selection, resulting in a more intensive utilisation of a group of highly preferred tools so that the global tool collection can be more compact. The changeover time for removing and mounting tool stations can be reduced accordingly.

The third strategy looks for possibilities to replace specific tools by similar ones that are already required by other bend lines in order to reduce the number of tool profiles in the final production setup. The similarities between the tools are judged by Euclidian distances between vectors representing their intrinsic geometric features in a multidimensional coordinate system [11]. An example of 2D clustering for similarity analysis used in this strategy can be seen in Figure 3.b. Tools with smaller distances to a specific tool are considered better candidates for replacement of that tool. Such replacement happens after a feasible tool assignment and bend sequence solution have been identified.

\section{IMPLEMENTATION}

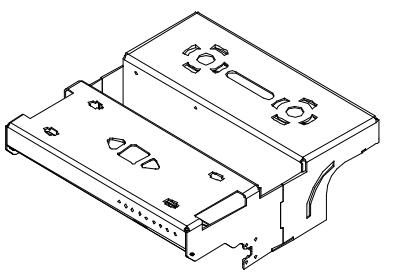

(a)

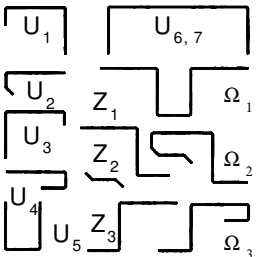

(c)

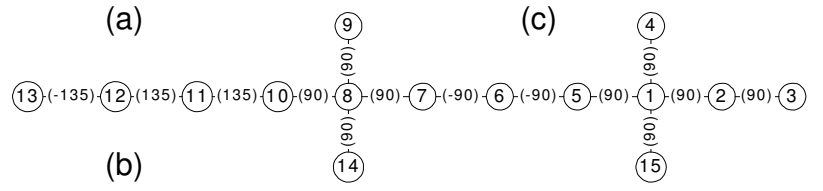

Figure 4: Case study a) design, b) abstraction, c) details. The step by step application of the proposed methodology is illustrated in this section by means of a case study. A part design as shown in Figure 4.a has been unfolded and the graph representation of the part was generated, as in Figure 4.b. Using this graph as the target graph, and the graph representations of the local details defined in Section 2.1 as pattern graphs, all the local details of the part have been matched, as shown in Figure 4.c. All the 
related collision patterns were verified to generate the constraints on geometric tool selection based on the rules established. These constraints are combined with technological constraints in order to preselect feasible tools for each bend line. Afterwards, tool preference factors were applied to eliminate tools with very low preferences. The result of this step served as input for the SCP algorithm (first optimisation strategy) to generate a feasible and optimised preselected tool set. This tool assignment was used as an initial selection for bend sequencing. A Branch-and-Bound search algorithm using heuristic penalties, as described in [2], was used for this purpose. In Figure 5, a typical search tree is shown with bend lines represented by nodes labelled according to the sequence of the search, and the penalty values indicated next to each node. Whenever a collision occurred at a bend line, as for nodes 1, 3, and 4, the initial tool selection for that bend line was refined. In that case, the original penalties were updated with extra tool change penalties calculated based on the preference factors used in the second optimisation strategy discussed in Section 2.2. The updated penalties are shown below the original penalties for collision nodes. Nodes with collisions unsolvable by changing tools were assigned infinite penalties in order to prune the remaining branches underneath, as is the case for node 1 . The updated penalties were then used to steer the search, in this case to node 3 . The same mechanism was used until a feasible bend sequence was found. At this point, the initial tool assignment had been replaced by a refined selection.

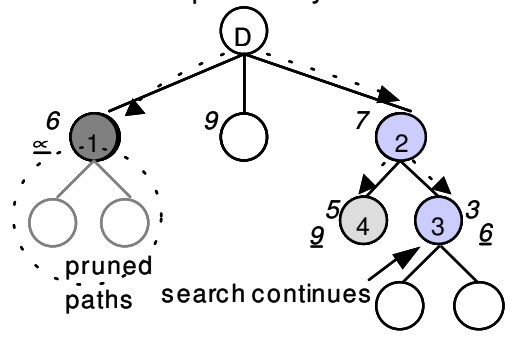

Figure 5: Refined selection during bend sequencing.

To further optimise the solution, the third optimisation strategy discussed in Section 2.2 was applied. For each bend line, the most similar tools for the feasible tool associated with that bend line were identified and verified for collisions when applied for the corresponding operation in the sequence. Therefore, this procedure identified all the tools compatible with the existing bend sequence, providing multiple tool-bend assignments that served as input for the first optimisation strategy. In this step, the total number of selected tools was minimised. Finally, the process plan was confirmed with the bend sequence and optimised tool solution found.

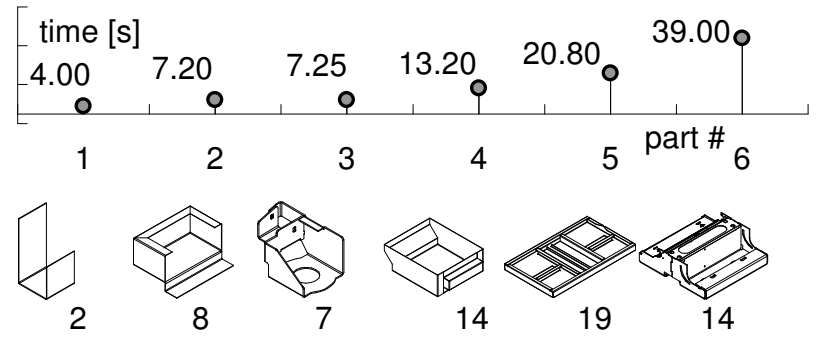

Figure 6: Performance record for test parts with indication of the number of bend features per part.

The described methodology has been implemented as part of an evaluative version of a commercial software solution. A series of test parts has been processed on a PC Pentium M 1.4GHz platform with 512MB RAM and Windows XP as OS. The performance of the system was recorded by the total processing time for finding feasible process plans, including bend sequencing and refined tool selection, as shown in Figure 6 . The automatic process planning was tested by several runs for each part, and the time recorded in seconds was averaged. It can be seen from the data that for all the test parts, feasible bend sequences were found within less than one minute with the tools automatically selected according to the geometry of the part.

\section{CONCLUSIONS}

In this paper a methodology was presented for tool selection in an integrated process planning context for bent sheet metal parts. Taking into account not only feasibility aspects of technology and geometry, but also production planning oriented guidelines, the method is able to deliver feasible and well-optimised tooling solutions, as result of a two phase procedure consisting of preselection and refined selection. Robust identification of feasible tools in both phases enhances a fast convergence towards a feasible process plan. The test results for an industrial software implementation have proven the feasibility of the methodology. Moreover, the performance of the system illustrates that the complexity of the algorithm is well under control.

\section{REFERENCES}

[1] Hoffmann, M., Geißler, U., Geiger, M., 1992, Computer-aided generation of bending sequences for die-bending machines, J. of Materials Processing Technology, 30/30, 1-12.

[2] Duflou, J., Kruth, J.-P., Van Oudheusden, D., 1999, Algorithms for the Design Verification and Automatic Process Planning for Bent Sheet Metal Parts, CIRP Annals, 48/1, 405-408.

[3] Márkus, A., Váncza, J. and Kovács, A., 2002, Constraint-Based Process Planning In Sheet Metal Bending, CIRP Annals, 51/1, 425-428.

[4] Shpitalni, M. and Radin, B., Critical Tolerance Oriented Process Planning in Sheet Metal Bending, 1999, Trans. of ASME J. of Mechanical Design, 121, 136-144.

[5] de Vin, L. J., de Vries, J. and Streppel, T., 2000, Process planning for small batch manufacturing of sheet metal parts, Int. J. Prod. Res., 38/17, 42734283.

[6] Franke, V., 1995, Automation of Tool Planning for Bent Components, Proc. of 3rd Int. Conf. on Sheet Metal, Birmingham, ISBN 0-9527664-0-X, 35-44.

[7] Singh, R. and Sekhon, G.S., 1999, An expert system for optimal selection of a press for a sheet metal operation, J. of Materials Processing Technology, 86/1-3, 131-138.

[8] Alva U., and Gupta, S. K., 2001, Automated Design of Sheet Metal Punches for Bending Multiple Parts in a Single Setup, Robotics and Computer-Integrated Manufacturing, 17/1-2, 33-47.

[9] Singh, R. and Sekhon, G. S., 2002, An intelligent system for optimal selection of dies and tools for sheet metal operations, J. Engineering Manufacture, 216/B, 821-828.

[10] Messmer, B.T. and H. Bunke, 1995, Subgraph Isomorphism in Polynomial Time, Tech. Report IAM 95-003, Univ. of Bern, Inst. of Computer Science and Applied Mathematics, Bern, Switzerland.

[11] Nguyen, T.H.M., Duflou, J., Kruth, J.-P. and Cattrysse, D., 2004, Tool Optimization for Sheet Metal Bending Operations, Proc. of $4^{\text {th }}$ CIRP Int. Sem. on Intelligent Computation in Manufacturing Eng., Sorrento, ISBN 88-87030-79-0, 257-262.

[12] El-Darzi, E., Mitra, G., 1995, Graph Theoretic Relaxations of Set Covering and Set Partitioning Problems, European J. of Operational Research, 87/15, 109-121. 